\title{
Avaliação de série de casos de eritema nodoso hansênico: perfil clínico, base imunológica e tratamento instituído nos serviços de saúde
}

\author{
Erythema nodosum leprosum case series report: clinical profile, immunological \\ basis and treatment implemented in health services
}

\author{
Jackeline Gomes Guerra ${ }^{1}$, Gerson Oliveira Penna ${ }^{2}$, Lia Cândida Miranda de Castro ${ }^{1}$, Celina \\ Maria Turchi Martelli ${ }^{3}$, Mariane Martins Araújo Stefani ${ }^{4}$ e Maurício Barcelos Costa ${ }^{5}$
}

\begin{abstract}
RESUM0
0 eritema nodoso hansênico é evento inflamatório agudo no curso crônico da hanseníase. É considerado evento de base imunológica e importante causa de morbidade e incapacidade física. Avaliou-se o perfil clínico, sorológico e histopatológico de 58 pacientes com eritema nodoso hansênico recrutados sequencialmente entre julho-dezembro de 2000, em área urbana hiperendêmica do Brasil Central (Estado de Goiás). A metade dos pacientes apresentava quadro reacional grave, e em $66 \%$ dos casos o primeiro episódio reacional ocorreu durante tratamento específico. A maioria dos casos com eritema nodoso hansênico e dos controles apresentaram reatividade para IgM anti-PGL I. Os achados histopatológicos mais freqüentes no eritema nodoso hansênico foram infiltrado neutrofílico, paniculite, vasculite e agressão neural. Dos pacientes com eritema nodoso hansênico, 96\% usaram corticosteróide sistêmico no primeiro episódio. Os casos de eritema nodoso hansênico estavam associados à neurite e raramente usaram talidomida como medicação isolada nos serviços de saúde.
\end{abstract}

Palavras-chaves: Hanseníase. Eritema nodoso hansênico. Anti-PGL I. Histopatologia. Tratamento.

\begin{abstract}
Erythema nodosum leprosum is an acute inflammatory event in the chronic course of leprosy. It is considered an immunological disorder and an important cause of morbidity and disability. We evaluate the clinical profile, serology and histhopathology 58 erythema nodosum leprosum patients sequentially recruited, from July- December 2000, in an endemic area in Central Brazil (Goiás State). Half of the reactins were considered severe and $66 \%$ of the cases had the first episode of reaction during specific treatment. The majority of patients and controls were positive to anti-PGL-I IgM. The more frequent histopathological findings in erythema nodosum leprosum were presence of intracellular acidfast bacilli, perivascular/peradnexial mononuclear inflammatory infiltrate, and neural aggression. Ninity six percent of the patients were treated with systemic steroid in the first episode. The results point out to the association between ENL and neuritis and the rare adoption of thalidomide as a solely medication in the health services.
\end{abstract}

Keys-words: Leprosy. Erythema nodosum leprosum. Anti-PGL I. Histopathology. Treatment.

As reações hansênicas são quadros inflamatórios agudos que podem ocorrer no curso da doença crônica hanseníase. A reação tipo 2 representa uma importante causa de morbidade e de incapacidade física em pacientes com hanseníase borderline lepromatosa (BL) e lepromatosa lepromatosa ( $\mathrm{LL})^{3}$, acometendo, principalmente, pacientes na faixa etária economicamente ativa ${ }^{16}$. A reação tipo 2 tem sido definida como: uma complicação imunológica da hanseníase multibacilar que se apresenta como nódulos eritematosos subcutâneos dolorosos que podem ulcerar.

\footnotetext{
1. Departamento de Medicina Tropical e Dermatologia do Instituto de Patologia Tropical e Saúde Pública da Universidade Federal de Goiás, Goiânia, G0. 2. Núcleo de Medicina Tropical da Faculdade de Medicina da Universidade de Brasília, Brasília, DF. 3. Departamento de Saúde Coletiva do Instituto de Patologia Tropical e Saúde Pública da Universidade Federal de Goiás, Goiânia, GO. 4. Departamento de Imunologia e Microbiologia do Instituto de Patologia Tropical e Saúde Pública da Universidade Federal de Goiás, Goiânia, G0. 5. Departamento de Patologia e Imagenologia da Faculdade de Medicina da Universidade Federal de Goiás, Goiânia, G0. Órgão Financiador: Projeto parcialmente financiado pela FUNAPE ㄲo 66.379.

Endereço para correspondência: Dra. Jackeline Gomes Guerra. Rua 17-A no 899/11ํandar, Setor Aeroporto, 74075-160 Goiânia, G0.

Tel: 62 229-4600; Fax: 62 212-6536

e-mail: jgguerra@ terra.com.br

Recebido para publicação em 01/12/03

Aceito em 17/6/2004
} 
Pode haver sinais de comprometimento sistêmico como febre, inflamação delinfonodos, neuropatia, envolvimento articular, testicular, ocular, de extremidades entre outros ${ }^{17} 40$.

0 tratamento dos episódios reacionais constitue-se em uma das principais prioridades no manejo do paciente com hanseníase para prevenção de incapacidades 1718192127 . Estes episódios não estão incluídos no Sistema de Notificação de Agravos Notificáveis ( SINAN) por não serem considerados eventos infecciosos associados à transmissão da infecção pelo $\mathrm{M}$ leprae. Desta forma, existem poucas informações disponíveis, nos serviços de controle, sobre 0 eritema nodoso hansênico (ENH), manifestação clínica mais freqüente da reação tipo 2 , com relação à freqüência, gravidade, impacto na rotina de atendimento médico, correlação com a terapia anti-hansênica e, particularmente, em relação à terapêutica instituída pelos serviços de saúde em nosso meio.

0 presente estudo apresenta aspectos epidemiológicos, clínicos, histopatológicos, sorológicos e terapêuticos de uma série de casos de hanseníase BL e $L$ com ENH, comparativamente ao grupo controle das mesmas formas clínicas sem ENH. Os resultados apresentados podem contribuir para o melhor entendimento do perfil dos pacientes e da instituição de sua terapêutica.

\section{PACIENTES E MÉTODOS}

Foram estudados 58 pacientes com hanseníase clínicahistologicamente classificados em BL/L com ENH e 32 BL L sem ENH entre agosto de 2000 a janeiro de 2001. Os pacientes foram recrutados seqüencialmente da demanda espontânea, dos maiores serviços de Dermatologia Sanitária do Estado de Goiás, Centro de Referência em Diagnóstico e Terapêutica e Hospital de Doenças Tropicais Anuar Auad, referência ambulatorial e hospitalar, respectivamente. Da série de casos com ENH, 14,8\% dos pacientes foram recrutados durante a internação hospitalar.

Critério de inclusão. Pacientes com diagnóstico clínico de ENH, considerando-se os seguintes sinais e sintomas: presença de nódulos eritematosos, calor local e, por vezes, dolorosos, associados a manifestações sistêmicas de intensidade variada, tais como: febre, calafrios, mal-estar, astenia, cefaléia, artralgias, adenopatias, mialgias ${ }^{16}$. Foram incluídos pacientes com ENH independente de episódios reacionais anteriores e do tratamento específico. Foram utilizados os critérios de classificação de gravidade dos quadros de ENH, propostos por Penna e cols: Leve: menos de 10 nódulos por segmento corporal comprometido, localizados mais freqüentemente nos membros inferiores, pouco dolorosos à palpação, sinais e sintomas sistêmicos ausentes ou de leve intensidade; Moderado: 10 a 20 nódulos por segmento corporal comprometido, necessariamente mais de um, dolorosos à palpação, associados a febre de moderada intensidade $(<38,40 \mathrm{C})$ com discreta sintomatologia sistêmica, podendo haver comprometimento da cadeia de linfonodos local e/ou regional; Grave: mais de 20 nódulos por segmento corporal comprometido, dolorosos espontaneamente, podendo haver lesões ulceradas, em geral envolvendo grande área do tegumento, acompanhados de expressiva sintomatologia sistêmica, como febre alta
( $>38,50 \mathrm{C})$, artralgias, calafrios, cefaléia importante, anorexia, fadiga, comprometimento generalizado de cadeia linfonoda $l^{16}$.

Pacientes BL/L sem história prévia de reação, doença imunossupressora e uso de terapêutica imunossupressora foram recrutados como grupo controle.

Aspectos clínicos. Todos os pacientes foram avaliados clinicamente pelo mesmo dermatologista no momento do recrutamento para caracterização clínica, dermatoneurológica, avaliação de incapacidades e classificação operacional, independente da rotina ambulatorial. A classificação da hanseníase em boderline ou lepromatosa obedeceu a correlacão dos parâmetros clínicos e histopatológicos, de acordo com Ridley e Joplin ${ }^{32}$.

Os dados de avaliação clínica do ENH foram coletados, de forma padronizada, de acordo com os seguintes aspectos ${ }^{16}$ : a) contagem das lesões de ENH por segmento corporal; b) localização das lesões por segmento corporal (cabeça e pescoço, tronco, membros superiores e inferiores); c) tipo de lesão elementar (nódulos, pústulas, úlceras); d) estágio inflamatório (agudo/ regressão); e) sintomatologia associada (febre, calafrios, artralgias, anorexia, orquite, lesões dolorosas e outros); e) classificação da gravidade do ENH (leve, moderado e grave).

0 grau de incapacidade física (GIF) e a classificação operacional em MB foram avaliados segundo as recomendações do Guia do Ministério da Saúde ${ }^{102}$.

No momento da triagem de pacientes foi realizada entrevista com coleta de dados sóciodemográficos, antecedentes familiares e pessoais detalhados e história clínica de início da hanseníase, através de questionário padronizado. Os dados sobre as consultas prévias por ENH com o seu respectivo tratamento foram coletados retrospectivamente da ficha/prontuário do paciente. A data do primeiro episódio reacional foi calculada tendo como referência à data do diagnóstico de hanseníase e foram agrupadas como: no momento do diagnóstico, durante os primeiros 6 meses de tratamento específico, do 70 ao 12을 mês, do $13^{\circ}$ ao $24^{0}$ mês e após a alta da poliquimioterapia (PQT).

Aspectos laboratoriais. Após a avaliação clínica os seguintes exames foram realizados:

a) Baciloscopia: 0 índice baciloscópico (IB) foi calculado pela média da contagem de bacilos álcool-ácido resistentes (BAAR) no tecido,colhidos em 4 locais (lóbulos de orelha, cotovelo direito e lesão de ENH, nos casos, e lesão BL ou LL, nos controles), corados pelo Ziehl-Nielsen e quantificados de 1 a $6+$; b) Histopatologia: A biópsia de pele foi coletada com punch número cinco, em lesão de paciente com ENH e, nos pacientes sem ENH em lesão característica de hanseníase BL e LL. Os tecidos foram rotineiramente fixados em formol tamponado a $10 \%$, processados em menos de 24 horas para corte em parafina e corados pela hematoxilina-eosina (HE) e Fite-Faraco. Todas as biópsias foram avaliadas por um único patologista com reconhecida experiência em dermatopatologia, da Faculdade de Medicina da UFG, sem conhecimento prévio sobre a caracterização clínica do paciente. As biópsias foram 
classificadas histopatologicamente de acordo com Ridley e Jopling ${ }^{32}$. Os seguintes critérios foram também avaliados: presença de vasculite na derme e/ou hipoderme, paniculite, infiltrado neutrofilico, presença de BAAR e agressão neural; c) Coleta de sangue para detecção de anticorpos anti-glicolipídico fenólico I (anti-PGL I): Foi realizada em um subgrupo de pacientes com ENH $(n=33)$ e 0 grupo de comparação ( $n=32)$. Amostras foram coletadas por venopunção, através de método vaccutainer em recipientes contendo EDTA, homogeinizadas, centrifugadas em período inferior a 24 horas e estocadas a $-20^{\circ} \mathrm{C}$, para detecção de anticorpos IgM anti- PGL I, pela técnica de ELISA. A detecção de anticorpos anti-PGL I foi realizada de acordo com metodologia utilizada e descrita anteriormente ${ }^{41}$, também de modo cego, no laboratório de Imunologia do Instituto de Patologia Tropical e Saúde Pública da UFG.

Aspectos éticos. 0 estudo foi aprovado pelo Comitê de Ética em Pesquisa da Universidade Federal de Goiás e os pacientes assinaram 0 Termo de Consentimento Informado Livre e Esclarecido de acordo com a Resolução 196 de 1996 do Conselho Nacional de Saúde.

Análise estatística. Foram realizadas análises descritivas de todas as variáveis clínicas, epidemiológicas e histopatológicas com as suas respectivas freqüências. Diferenças de frequêencia foram avaliadas pelo teste de quiquadrado e comparação entre médias pelo teste $t$, considerando-se 0 p <0,05 como significância estatística. 0 gráfico de caixa foi utilizado como análise exploratória para avaliação da sorologia anti- PGLI entre pacientes com ENH e seu grupo de comparação. 0 gráfico de dispersão foi construído para avaliar os níveis de anticorpos anti-PGL I e IB, no momento da consulta, bem como o cálculo do coeficiente de correlação ( $r$ ) entre essas variáveis. A análise de dados foi realizada através do programa SPSS, versão 4.0.

\section{RESULTAD0S}

Noventa pacientes de hanseníase foram incluídos, sendo 58 casos ( ENH) e 32 controles ( sem ENH). A média de idade dos pacientes com ENH foi de 34,5 anos $(d p=11,9)$ e dos controles foi 38,7 ( $d p=13,7)$, diferença não estatisticamente significante $(p=0,3)$. Aproximadamente, $60 \%$ dos pacientes com ENH e controles eram do sexo masculino, não havendo diferença estatísticamente significante $\left(X^{2}=0,10: p=0,7\right)$. Quanto aos casos, 41 (70,7\%) eram apenas alfabetizados. A maioria (69\%) deles residia em Goiânia, aproximadamente a metade referiu contato familiar com caso de hanseníase e 87,9\% não apresentaram cicatriz vacinal de BCG ( Tabela 1).

Em relação às características dos pacientes com ENH, a forma clínica lepromatosa foi predominante (87,9\%) e 15,5\% dos pacientes apresentaram algum grau de incapacidade. № momento do diagnóstico da hanseníase $60,4 \%$ apresentaram IB $>2$ enquanto ao diagnóstico do ENH apenas 29,3\% apresentaram altos IB. Dos pacientes, 20,7\% apresentavam lesões em todos os segmentos do corpo, sendo que $86,2 \%$ e $77,6 \%$ apresentavam lesões nos membros superiores e
Tabela 1 - Características sociodemográficas dos pacientes diagnosticados com ENH, Goiânia, Goiás.

\begin{tabular}{lrc}
\hline Variáveis & Número & Porcentagem \\
\hline Sexo & 34 & 58,6 \\
$\quad$ masculino & 24 & 41,4 \\
$\quad$ feminino & & \\
Escolaridade & 41 & 70,7 \\
$\quad$ analfabeto/alfabetizado & 17 & 29,3 \\
$\quad$ 10 Grau/2º Grau $^{\text {Residência }}$ & & \\
$\quad$ Goiânia e aglomerado & 40 & 69,0 \\
$\quad$ outros municípios/Estados & 15 & 31,0 \\
Antecedente familiar de MH & & \\
$\quad$ sim & & 53,4 \\
$\quad$ não & 31 & 39,7 \\
Cicatriz vacinal de BCG & 23 & 12,1 \\
$\quad$ sim & & 87,9 \\
$\quad$ não & 7 & \\
\hline
\end{tabular}

MH: Hanseníase; BCG: bacilo de Calmet Guerrin

${ }^{*} 4$ pacientes sem informação

membros inferiores, respectivamente. Quanto ao momento do primeiro episódio de ENH, em $66 \%$ dos pacientes manifestou-se durante 0 curso do tratamento (Tabela 2). Segundo os critérios de gravidade, os pacientes do presente estudo foram classificados como: 34,5\% ENH leve, 13,8\% ENH moderado e 51,7\% ENH grave.

0 número de atendimentos médicos por paciente com episódio reacional, no período estudado, foi em média 4,5, variando de 1 a 23 visitas. Com relação às características morfohistológicas das biópsias de pele, 12 pacientes com ENH apresentaram todos os critérios histopatológicos simultaneamente, sendo a presença de BAAR $(91,4 \%) \quad 0$ achado mais constantes (Tabela 3).

A análise univariada não demonstrou diferença estatisticamente significante entre biópsias de pele de pacientes com ENH e pacientes sem ENH em relação às formas clínicas e a presença de BAAR. Houve diferença estatisticamente significante quanto à presença de neutrófilos, paniculite, agressão neural e vasculite nos pacientes com ENH quando ao grupo controle (Tabela 4).

Quanto ao tempo de aparecimento do primeiro episódio reacional em relação ao diagnóstico de hanseníase, 17\% dos ENH foram diagnosticados no momento do primeiro episódio reacional, em $54,7 \%$ dos casos 0 evento ocorreu no primeiro ano da PQT, sendo que em $66 \%$ dos pacientes o ENH manifestou-se durante os 24 meses de tratamento. Em 17\% dos casos de ENH ocorreu após o término da PQT (Figura 1).

A Figura 2 apresenta a distribuição da densidade ótica da sorologia anti-PGL I nos pacientes com ENH e controles. Os resultados indicam que a mediana dos valores nos pacientes MB sem ENH foi de 0,83 versus mediana de 0,23 nos pacientes com ENH, diferença não estatisticamente significante.

0 gráfico de dispersão na Figura 3 mostra a correlação dos resultados do índice baciloscópico e níveis de IgM anti- 
Tabela 2 - Características clínicas dos pacientes diagnosticados com ENH, Goiânia, Goiás.

\begin{tabular}{|c|c|c|}
\hline Variáveis & Número & Porcentagem \\
\hline \multicolumn{3}{|l|}{ Forma clínica } \\
\hline lepromatosa & 51 & 87,9 \\
\hline boderline & 7 & 12,1 \\
\hline \multicolumn{3}{|l|}{ Índice baciloscópico no diagnóstico de $\mathrm{MH}^{\mathrm{a}}$} \\
\hline$\leq 1$ & 15 & 28,3 \\
\hline $1,1-2,0$ & 6 & 11,3 \\
\hline $2,1-3,0$ & 15 & 28,3 \\
\hline$\geq 3,1$ & 17 & 32,1 \\
\hline \multicolumn{3}{|l|}{ Índice baciloscópico atual } \\
\hline$\leq 1$ & 26 & 44,8 \\
\hline $1,1-2,0$ & 15 & 25,9 \\
\hline $2,1-3,0$ & 6 & 10,3 \\
\hline$\geq 3,1$ & 11 & 19,0 \\
\hline \multicolumn{3}{|l|}{ Grau de incapacidade final } \\
\hline 0 & 49 & 84,5 \\
\hline 1 & 7 & 12,1 \\
\hline 2 & 2 & 3,4 \\
\hline \multicolumn{3}{|l|}{ Área corporal acometida } \\
\hline disseminado ${ }^{\mathrm{b}}$ & 12 & 20,7 \\
\hline tronco, membros superiores e inferiores & 8 & 13,8 \\
\hline membros superiores e inferiores & 11 & 19,0 \\
\hline membros superiores $^{c}$ & 50 & 86,2 \\
\hline membros inferiores ${ }^{c}$ & 45 & 77,6 \\
\hline \multicolumn{3}{|c|}{ Tempo de tratamento específico no $1^{0}$ episódio de ENH } \\
\hline diagnóstico de MH & 9 & 17,0 \\
\hline$\leq 6$ meses de $\mathrm{PQT}$ & 16 & 30,2 \\
\hline 7 - 12 meses de PQT & 13 & 24,5 \\
\hline 13 - 24 meses de PQT & 6 & 11,3 \\
\hline pós-alta de PQT & 9 & 17,0 \\
\hline \multicolumn{3}{|l|}{ Tratamento do primeiro episódio de ENH } \\
\hline prednisona & 31 & 53,4 \\
\hline corticosteróide injetável & 14 & 24,2 \\
\hline prednisona e talidomida & 11 & 19,0 \\
\hline talidomida & 2 & 3,4 \\
\hline
\end{tabular}

MH: Hanseníase; ENH: eritema nodoso hansênico; PQT: poliquimioterapia

a 5 pacientes sem informação

${ }^{\mathrm{b}}$ Nódulos localizados em todos os segmentos corporais

c Percentuais não correspondem a 100\%

Tabela 3 - Características morfohistológicas de biópsias de pele de 58 pacientes diagnosticados com ENH, Goiânia, Goiás.

\begin{tabular}{lccccc}
\hline & A & B & C & D & E \\
\hline BAAR & + & + & + & + & + \\
Neutrófilos & - & + & + & + & + \\
Paniculite & - & - & + & + & + \\
Agressão neural & - & - & - & + & + \\
Vasculite & - & - & - & - & + \\
\hline Total & $53(91,4 \%)$ & $45(77,6 \%)$ & $41(70,7 \%)$ & $33(56,9 \%)$ & $12(22,4 \%)$ \\
\hline A
\end{tabular}

$\mathrm{A}=$ presença de BAAR

$B=$ presença de BAAR e neutróilios

$\mathrm{C}=$ presença de $\mathrm{BAAR}$, neutrófilos e paniculite

$\mathrm{D}=$ presença de BAAR, neutróilos, paniculite e agressão neural

$\mathrm{E}=$ presença de BAAR, neutrófilos, paniculite, agressão neural e vasculite.
Tabela 4 - Característicasmorfohistológicas de biópsias de pele depacientes com ENHe controles multibacilares, Goiânia, Goiás.

\begin{tabular}{|c|c|c|c|c|}
\hline Variáveis & $\begin{array}{c}\text { ENH } \\
(\mathrm{N}=58)\end{array}$ & $\begin{array}{c}\text { Controles } \\
(\mathrm{N}=32)\end{array}$ & $\begin{array}{c}\text { Teste de } \\
\chi^{2}\end{array}$ & (valor de p) \\
\hline \multicolumn{5}{|l|}{ Forma clínica } \\
\hline $\mathrm{BL}$ & $5(8,6)$ & $6(18,7)$ & & \\
\hline $\mathrm{L}$ & $53(91,4)$ & $26(81,3)$ & 0,01 & $(\mathrm{P}=0,91)$ \\
\hline \multicolumn{5}{|c|}{ Características histológicas } \\
\hline \multicolumn{5}{|l|}{ BAAR } \\
\hline positivo & $53(91,4)$ & $30(93,8)$ & & \\
\hline negativo & $5(8,6)$ & $2(6,2)$ & 0,16 & $(\mathrm{P}=0,69)$ \\
\hline \multicolumn{5}{|l|}{ neutrófilos } \\
\hline presente & $45(77,6)$ & $4(12,5)$ & & \\
\hline ausente & $13(22,4)$ & $28(87,5)$ & 35,2 & $(\mathrm{P}=0,001)$ \\
\hline \multicolumn{5}{|l|}{ paniculite ${ }^{\mathrm{a}}$} \\
\hline presente & $51(87,9)$ & $13(40,6)$ & & \\
\hline ausente & $6(10,3)$ & $19(59,4)$ & 9,1 & $(\mathrm{P}=0,001)$ \\
\hline \multicolumn{5}{|c|}{ agressão neural ${ }^{b}$} \\
\hline presente & $48(82,8)$ & $16(50,0)$ & & \\
\hline ausente & $10(17,2)$ & $15(46,9)$ & 9,1 & $(\mathrm{P}=0,001)$ \\
\hline \multicolumn{5}{|l|}{ vasculite } \\
\hline presente & $18(31,0)$ & 2 & $(6,2)$ & \\
\hline ausente & $40(69,0)$ & $30(93,8)$ & 9,3 & $(\mathrm{P}=0,001)$ \\
\hline
\end{tabular}

BL: borderline lepromatosa; $\amalg$ : lepromatosa lepromatosa

a 1 biópsia de ENH sem tecido subcutâneo representado

${ }^{b} 1$ de controle sem filete nervoso encontrado

PGL I estratificados por pacientes com ENH e pacientes com hanseníase MB sem ENH. Não observamos correlação entre 0 IB e 0 nível de anticorpos IgM anti-PGL I nos pacientes com ENH $(p>0,05)$, quando comparados aos controles sem $\mathrm{ENH}(\mathrm{p}<0,01)$.

\section{DISCUSSÃ0}

A talidomida continua sendo a droga de escolha para tratar 0 ENH, apesar da recente controvérsia gerada pelo posicionamento contrário ao uso dessa droga pela Organização Mundial da Saúde 2630 . Em nosso estudo, chama a atenção que menos que $5 \%$ dos pacientes com ENH receberam talidomida e a restrição de uso não foi 0 gênero, pois homens foram predominantes na casuística. Amaioria dos pacientes foi tratada com corticosteróides cujo uso continuado apresenta efeitos colaterais importantes. 0 uso de corticosteróide na quase totalidade $(96,6 \%)$ dos pacientes tem sido justificado pela associação freqüente entre ENH e neurite ou alguma outra indicação absoluta do uso de corticosteróide ${ }^{29}$, conforme descrito anteriormente ${ }^{28}$.

0 tratamento de eleição dos quadros moderados e graves de ENH preconizado pelo Ministério da Saúde é a talidomida, exceto nas seguintes situações: quadro de ENH necrotizante, neurite, orquiepididimite, irite, nefrite, mão e pé reacional, quando está precisamente indicado o uso de corticosteróides sistêmicos. 0 uso da talidomida em mulheres em idade fértil foi recentemente revisto no texto da Lei $10.651^{4}$. Os quadros leves podem ser tratados com antiinflamatórios não esteróide (AINES). Dentre os efeitos colaterais da talidomida destacase a neuropatia periférica, sonolência, obstipação intestinal, 


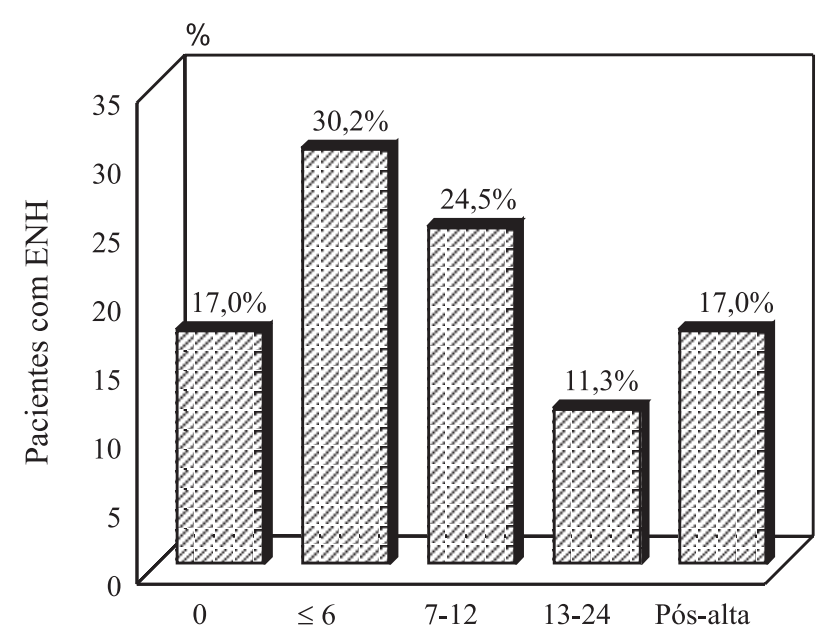

Duração do tratamento (meses)

Figura 1 - Relação entre o primeiro episódio de ENH e o tempo de tratamento para hanseníase.

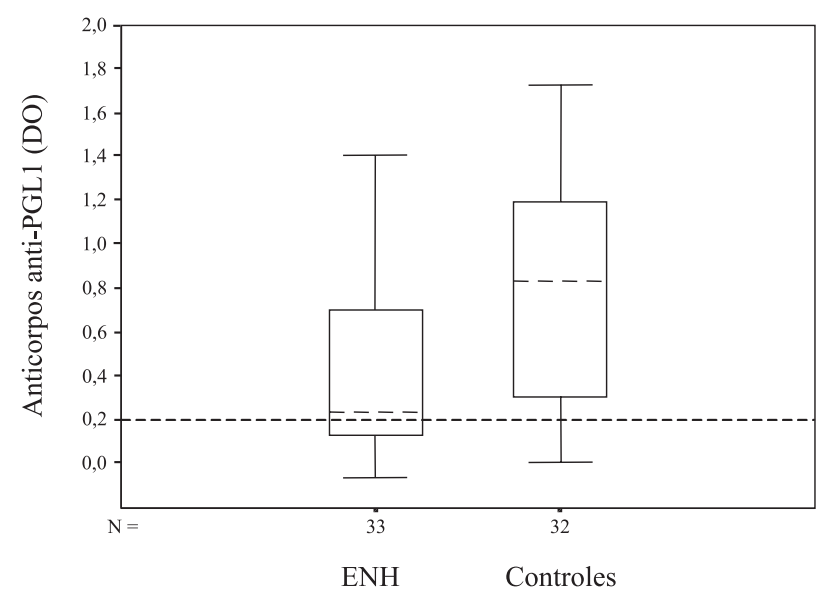

Alinha superior e inferior da caixa são os percentis 25 e 75, e 0 comprimento da caixa 0 intervalo interquartílico (IQR). Alinha pontillhada que corta a caixa é 0 valor da mediana. Abarra de erro superior representa a maior observação igual ao percentil 75 mais 1,5 x IQR. A barra de erro inferior é a menor observação do percentil 25 menos 1,5 x IQR. A linha pontilhada que corta todo o gráfico corresponde ao ponto de corte de positividade dos anticorpos anti-PGL1 ( $\left.\mathrm{DO}^{3} 0,2\right)$.

Figura 2 - Níveis de anticorpos anti-PGL I em 33 pacientes ENH e 32 pacientes com hanseníase BL e L.

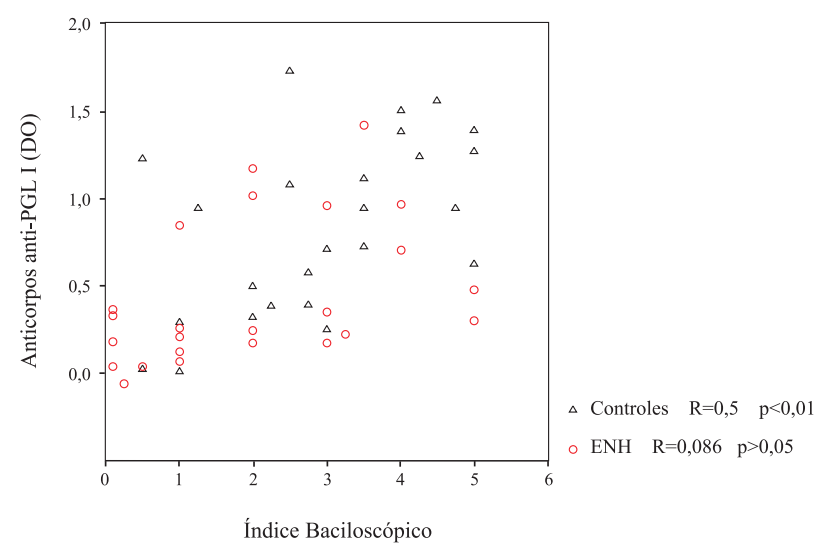

Figura 3. Correlação entre índice baciloscópico e níveis de anticorpos anti-PGL I, estratificado por 33 pacientes com ENH e 32 pacientes com hanseníase BL e $L$ sendo que teratogenicidade é a responsável pelo estigma no seu uso ${ }^{16}$. Os corticosteróides quando utilizados em altas doses e/ou por tempo prolongado podem levar a hipertensão arterial, osteoporose, gastrite, úlcera péptica e agravamento de infecções latentes ${ }^{10}$.

Os pacientes com ENH do presente estudo eram predominantemente $\amalg$ ( $81 \%$ ) em consonância com a literatura ${ }^{317}$ que descreve essa forma como importante fator de risco para 0 desenvolvimento de $\mathrm{ENH}^{20}{ }^{36}$. Embora esse evento possa ser mais freqüente no sexo masculino, talvez pela maior prevalência de formas $\amalg$ nos homens ${ }^{53}{ }^{34}$, alguns estudos têm mostrado que 0 gênero não é fator predisponente ${ }^{2025} 36$. Outras investigações têm mostrado que as reações hansênicas são mais freqüentes entre 20 e 40 anos de idade ${ }^{71539}$. Idade inferior a 40 anos foi considerada fator de risco no Nepal para o desenvolvimento de $\mathrm{ENH}^{20}$, ao contrário dos estudos de Nery e cols ${ }^{25}$ e Saunderson e $\mathrm{cols}^{36}$.

Um número expressivo de pacientes apresentava $\mathrm{IB}>2$ no momento do diagnóstico de hanseníase $(60,4 \%)$ e IB $<2$ no momento atual da avaliação do $\mathrm{ENH}(70,7 \%)$, provavelmente pela negativação do IB promovida pelo tratamento específico e até mesmo pelos episódios reacionais. Manandhar e cols ${ }^{20}$ observaram em seu estudo que IB elevado (>4) no início do tratamento constitue em fator de risco para 0 desenvolvimento de $\mathrm{ENH}^{20}$. Rodrigues e cols ${ }^{33}$ em investigação realizada no Brasil evidenciaram correlação positiva entre o IB no momento do diagnóstico e 0 número de episódios durante 0 tratamento e após a alta por cura ${ }^{33}$.

Dentre os pacientes com ENH deste estudo, 20,7\% tinham lesões disseminadas por todos os segmentos corporais, sendo que $86,2 \%$ e 77,6\% tinham lesões nos membros superiores e inferiores, respectivamente, que são os locais mais freqüentemente acometidos, evidenciando a característica clínica do ENH e a necessidade de classificação padronizada de gravidade dos casos.

$0 \mathrm{ENH}$ pode ocorrer em qualquer momento durante a evolução da hanseníase, sobretudo durante 0 tratamento específico, que leva à morte bacilar e liberação maciça de antígenos micobacterianos e conseqüentemente à formação dos imunecomplexos envolvidos na sua fisiopatologia ${ }^{13}$. A maioria dos pacientes apresentou o primeiro episódio reacional durante 0 tratamento específico. Estes resultados são concordantes com outros estudos 203637 e indicam que os pacientes devem ser cuidadosamente monitorizados e orientados quanto ao risco de desenvolver ENH, não só durante 0 tratamento específico, mas também após a alta por cura da hanseníase. Esse último dado reveste-se de importância na medida em que se discute a redução do tempo de tratamento de 12 para 6 meses. Embora a ocorrência do estado reacional não pareça estar relacionada à terapêutica nem ao tempo de tratamento ${ }^{19} 22350$ paciente deve ser alertado durante seu tratamento da possibilidade de aparecimento dos estados reacionais e da necessidade de, nesses casos, procurar o serviço de saúde.

Aproximadamente a metade dos pacientes com hanseníase desenvolverá pelo menos 1 episódio reacional durante 0 tratamento específico ${ }^{14}$. Scollard e cols ${ }^{38}$ em estudo 
prospectivo que acompanhou 176 pacientes MB recémdiagnosticados por pelo menos 3 anos após o início da PQT, detectaram que $0 \mathrm{ENH}$ esteve associado com recorrências freqüentes ${ }^{38}$. 0 número médio de atendimentos por paciente com ENH, no presente estudo, foi de 4,5, 0 que mostra que 0 ENH é importante causa de atendimentos médicos nos serviços públicos em nosso meio.

Houve predomínio de pacientes com ENH sem cicatriz vacinal da BCG ( 87,9\%) , o que pode ser devido ao fato dessa vacina inicialmente ter sido administrada por via oral e apenas a partir de 1973 por via intradérmica, deixando cicatriz. Quanto ao grau de incapacidade física, 15,5\% dos pacientes apresentou algum GIF, possivelmente refletindo diagnóstico tardio da hanseníase ${ }^{1129}$.

Dos pacientes com ENH deste estudo de série de casos, 54,5\% apresentaram sorologia positiva para anticorpos IgM anti-PGL I, entretanto com concentração inferior quando comparados com os pacientes com hanseníase BB e LLsem reação. Esses resultados são concordantes com aqueles encontrados por Cho e cols ${ }^{6}$ que observaram que os niveis de anticorpos anti-PGL I são menores em pacientes com ENH que em pacientes com hanseníase $L$ sem $\mathrm{ENH}^{6}$. Andreoli e cols ${ }^{1}$ monitorizaram os níveis desses anticorpos antes, durante e após o episódio de ENH em 12 pacientes $\amalg$ e observaram que durante 0 episódio reacional houve uma queda das concentrações, que retornaram para os níveis pré-reacionais após o restabelecimento desses pacientes. Os autores salientam que 0 motivo para essa diminuição é desconhecido, mas que pode ser devido à formação e deposição de imunecomplexos nos tecidos, de acordo com a fisiopatologia do $\mathrm{ENH}^{1}$.

Mais recentemente, tem sido demonstrado que durante 0 surto de ENH os pacientes $L$, que apresentam perfil de resposta imune humoral (TH2), desenvolvem ativação transitória da resposta imune celular (TH1), podendo levar à inibição da resposta TH2 e, conseqüentemente, diminuição da produção de anticorpos, 0 que poderia justificar essa alteração nos níveis dos anticorpos durante 0 curso do quadro de $\mathrm{ENH}^{24}$.

É geralmente aceito que o índice baciloscópico é fator determinante dos níveis de anticorpos anti-PGL I. Em estudo anterior tipo caso-controle que comparou pacientes hansenianos recém-diagnosticados com reação tipo 1 e 2 com controles normais pareados por índice baciloscópico, os pacientes com hanseníase, independente do seu estado reacional, apresentaram altos níveis de IgM anti-PGL I, mostrando que o perfil desses anticorpos está diretamente associado $\mathrm{com} 0 \mathrm{IB}^{41}$. No presente estudo quando correlacionou-se a sorologia do anticorpo anti- PGL I com 0 índice baciloscópico, os pacientes com ENH não apresentaram correlação positiva $(p>0,05)$, diferente dos pacientes MB sem ENH $(p<0,01)$, 0 que pode estar relacionado com a não uniformidade da amostra que incluiu pacientes em diferentes momentos do tratamento específico, diferentes IB e uso ou não de drogas anti-reacionais.

No presente estudo, os achados mais constantes ao exame histopatológico das lesões de ENH foram: paniculite e agressão neural $(79,3 \%)$ e infiltrado neutrofilico $(77,6 \%)$, e a vasculite esteve presente em 31\% dos casos. Entretanto, todas essas alterações foram estatisticamente significantes quando as biópsias de ENH foram comparadas às biópsias de lesão de hanseníase BLe LL sem ENH. Portanto, de acordo com a literatura, 0 achado histopatológico mais característico do ENH é 0 infiltrado neutrofílico ${ }^{8}$, e 0 presente estudo mostrou que paniculite, agressão neural e vasculite, assim como 0 infiltrado de neutrófilos, também caracterizam essa afecção. As variações histopatológicas encontradas nas lesões de ENH podem ser devidas às diferenças nos pacientes biopsiados como, por exemplo, o tempo de evolução da lesão no momento da biópsia.

Histopatologicamente, o ENH é caracterizado pelo influxo de neutrófilos entre os histiócitos espumosos repletos de bacilos, através da derme e, às vezes, da hipoderme, exceto em estágios mais tardios em que predominam os linfócitos, sendo que a vasculite de pequenos vasos é encontrada em cerca de metade dos caso ${ }^{31}$. Segundo alguns autores, 0 ENH caracteriza-se basicamente por ser uma vasculite leucocitoclástica neutrofílica ocasionalmente entremeada por eosinófilos ${ }^{9}$. Murphy e cols ${ }^{23}$ estudaram biópsias de pele de quatro pacientes com ENH e observaram vasculite necrotizante em todas elas, sendo que nas lesões agudas havia infiltrado neutrofílico e nas lesões mais antigas linfócitos e plasmócitos ${ }^{23}$. Sehgal e cols $\mathrm{s}^{39}$ estudaram biópsias de 17 casos de ENH e em todas elas encontraram o predomínio do infiltrado neutrofílico ${ }^{39}$.

Os nossos achados evidenciaram o uso frequente de corticosteróides sistêmico e a ausência de talidomida como medicação isolada no tratamento do ENH em serviços de saúde. Estes dados apontam para a gravidade dos quadros clínico de ENH e para a freqüente associação com neurite. 0 presente estudo alerta para a necessidade de padronização das intervenções de acordo com a classificação clínica do ENH, bem como para 0 monitoramento adequado para prevenção de incapacidades ${ }^{26}$.

\section{REFERÊNCIAS BIBLIOGRÁFICAS}

1. Andreoli A, Brett SJ, Draper P, Payne SN, Rook GAW. Changes in -circulating antibody levels to the major phenolic glycolipid during erythema nodosum leprosum in leprosy patients. International Journal of Leprosy 53:211217, 1951.

2. Becx-Bleumink M, Berhe D. Occurrence of reactions, their diagnosis and management in leprosy patients treated with multidrug therapy; experience in the leprosy control of the all Africa leprosy and rehabilitation training center (ALERT) in Ethiopia. International Journal of Leprosy 60:173-183, 1992.

3. Bleharski JR, Li H, Meinken C, Graeber TG, Ochoa MT, Yamamura M, Burdick A, Sarno EN, Wagner M, Röllinghoff M, Rea TH, Colonna M, Stenger S, Bloom BR, Eisenberg D, Modlin RL. Use of genetic profiling in leprosy to discriminate clinical forms of the disease. Science 301: 1527-1530, 2003.

4. Brasil. Ministério da Saúde. Diário Oficial da União, Lei 10.651 de 16 de abril de 2003, sessão 1, página 25, Brasília, DF, 17 de abril de 2003.

5. Browne SG. Erythema nodosum in leprosy. Journal of Chronic Diseases 16:23-30, 1963.

6. Cho SN, Fukiwara T, Hunter SW, Rea TH, Gelber RH, Brennan PJ. Use of an artificial antigen containing the 3,6-di-O-methyl â-D-glucopranosyl epitope 
for the serodiagnosis of leprosy. Journal of Infectious Diseases 50:311322, 1984.

7. Chopra NK, Agrawal JS, Pandya PG. Reactions in leprosy - A study of 250 patients in a multidrug therapy project, Baroda District, Gurajat, Índia. International Journal of Dermatology 29:490-493, 1990.

8. Lucas S. Bacterial disease. In: Elder D, Elenitsas R, Jaworsky C, Johnson JB (eds) Lever's histopathology of the skin, $8^{\text {th }}$ edition, Lippincott-Raven, Philadelphia, p. 457-502, 1997.

9. Fleury RN. Manifestações sistêmicas. In: Talhari S, Neves RG (eds) Hanseníase, 2a edição, Gráfica e Editora Tropical, Manaus, p.73-77, 1997.

10. Fundação Nacional de Saúde. Manual de Prevenção de Incapacidades. Ministério da Saúde, Brasília, 1997.

11. Fundação Nacional de Saúde. Hanseníase no Brasil, progressos e dificuldades em relação à eliminação. Ministério da Saúde, Brasília, 1998.

12. Gallo MEN, Alvin MFS, Nery JAC, Albuquerque ECA, Sarno EN. Two multi drug fixed-dosage treatment regimens with multibacillary leprosy patients. Indian Journal of Leprosy 68: 235-245, 1996.

13. Gillis TP, Krahehbuhl JL. Global elimination of leprosy. Reviews in Medical Microbiology 9:39-48, 1998.

14. Gillis W. Leprosy at a turning point?. International Journal of Leprosy $68: 312-318,2000$

15. Groenen G, Janssens L, Kayembe T, Nollet E, Coussens L, Pattyn SR. Prospective study on the relationship between intensive bactericidal therapy and leprosy reactions. International Journal of Leprosy 54:236-244, 1986.

16. Guerra JG, Penna GO, Castro LCM, Martelli CMT, Stefani MMA. Eritema nodoso hansênico: Atualização clínica e terapêutica. Anais Brasileiros de Dermatologia 77:389-407, 2002.

17. International Leprosy Association. Report of the - Technical Forum - Paris, France, 22 - 28 February 2002. International Journal of Leprosy 70:S1-S62, 2002.

18. Jacobson RR, Krahenbuhl JL. Leprosy. Lancet 353:655-660, 1999.

19. Lockwood DN. Leprosy elimination-a virtual phenomenon or a reality? British Medical Journal 324: 1516-1518, 2002.

20. Manandhar R, LeMaster JW, Roche P. Risk factors for erythema nodosum leprosum. International Journal of Leprosy 67:270-278, 1999.

21. Martelli CMT, Stefani MMA, Penna GO, Andrade AL. Endemias e epidemias brasileiras, desafios e perspectivas de investigação científica: hanseníase. Revista Brasileira de Epidemiologia 5:273-285, 2002.

22. Moraes MO, Duppre NC, Suffys PN, et al. Tumor necrosis factor-a promoter polimorphism TNF-a is associated with a stronger delayed-type hypersensivity reaction in the skin of borderline tuberculoid leprosy patients. Immunogenetics 53:45-47, 2001

23. Murphy GF, Sanchez NP, Flynn TC, Sanchez JL, Mihm Jr MC, Soter NA. Erythema nodosum leprosum: Nature and extent of the cutaneous microvascular alterations. Journal of American Academy of Dermatology 14:59-69, 1986

24. Nath I, Vemuri N, Reddi AL, Jain S, Brooks P, Colston MJ, Misra RS, Ramesh V. The effect of antigen presenting cells on the cytokine profiles of stable and reactional lepromatous leprosy patients. Immunology letters 75:6976,2000

25. Nery JC, Viera LM, Matos HJ, Gallo ME, Sarno EN. Reactional states in multibacillary Hansen disease patients during multidrug therapy. Revista do Instituto de Medicina Tropical de São Paulo 40:363-370, 1998.

26. Pannikar V. The Return of Thalidomide: New Uses and Renewed Concerns. Available at: http://who-umc.org. Penna G0, Pereira GFM, Talhari S. On Thalidomide and WHO Policies. Available at: Salvatore Leprosy Net noto@ cefpas.it. Lockwood D, Bryceson A. The Return of Thalidomide - a reply. Available at: Salvatore Leprosy Net - noto@ cefpas.it, 2003.

27. Penna GO. A propósito da eliminação da hanseníase no Brasil. Paramédicos 1: $40-44,2002$

28. Penna G0. Hanseníase e 0 uso da talidomida no eritema nodoso hansênico: perspectivas de pesquisas. Tese de Doutorado, Universidade de Brasília, Brasília, DF, 2002.

29. Penna G0, Pereira G, Moreira M. Guia Para 0 Controle da Hanseníase Cadernos da Atenção Básica no 10, Ministério da Saúde, Brasília, 2002.

30. Penna G0, Pinheiro AMC, Hajjar LA. Talidomida: mecanismo de ação, efeitos colaterais e uso terapêutico. Anais Brasileiros de Dermatologia 73: 501-517, 1998.

31. Ridley DS, Job CK. The pathology of leprosy. In: Hastings RC (ed) Leprosy, $1^{\text {st }}$ edition, Churchill-Livingstone, Edinburgh, p.100-133, 1985.

32. Ridley DS, Jopling DS. Classification of leprosy according to immunity. A five-group system. International Journal of Leprosy 34: 255-273, 1966.

33. Rodrigues ALP, Almeida AP, Rodrigues BF, Pinheiro CA, Borges DS, Mendonça MLH, Silva VEF, Goulart IMB. Occurrence of late lepra reaction in leprosy patients: subsidies for implementation of a specific care program. Hansenologia Internationalis 25:7-16, 2000.

34. Sampaio SAP, Rivitti EA, Marchese LMT. Hanseníase. In: Sampaio SAP, Rivitti EA(ed) Dermatologia, 2a edição, Artes Médicas, São Paulo, p.467-488, 2000.

35. Santos AR, Almeida AS, Suffys PN, Moraes MO, Mattos HJ, NeryJAC, Cabello PH, Sampaio EP, Sarno EN. Tumor Necrosis Factor Promoter Polymorphism (TNF-a) seems to protect Against Development of Severe Forms of Leprosy in a Pilot Study in Brazilian Patients. International Journalof Leprosy 68: $325-327,2000$

36. Saunderson P, Gebre S, Byass P. ENL reactions in the multibacillary cases of the AMFES cohort in central Ethiopia: incidence and risk factors. Leprosy Review 71:318-324, 2000.

37. Schreuder PAM. The occurrence of reactions and impairments in leprosy: experience in the leprosy control program of three provinces in northeastern Thailand, 1978-1995. II. Reactions. International Journal of Leprosy 66:159-169, 1998.

38. Scollard DM, Smith T, Bhoopat L, Theetranont C, Rangdaeng S, Morens DM. Epidemiological characteristics of leprosy reactions. International Journal of Leprosy 62: 559-567, 1994.

39. Sehgal VN, Sharma V. Reactions in Leprosy - A prospective study of clinical, bacteriological, immunological and histopathological parameters in thirtyfive Indians. Journal of Dermatology 15: 412-419, 1988.

40. Smith C, Nocholl P, Brakel WW, Lockwood D, Naafs B, Scollard D, Saunderson P. Special workshop on Repeated and Late Reactions. International Journal of Leprosy 70: 339-341, 2002.

41. Stefani MMA, Martelli CMT, Morais-Neto OL, Martelli P, Costa MB, Andrade ALSS. Assessment of anti-PGL I as a prognostic marker of leprosy reaction. International Journal of Leprosy 66: 356-364, 1988. 